\section{Atividade física insuficiente: fatores associados e qualidade de vida}

\section{Insufficient physical activity: factors associeted and quality of life}

\author{
Maryane Oliveira-Campos ${ }^{1}$ \\ Marcos Gonçalves Maciel ${ }^{2}$ \\ João Felício Rodrigues Neto ${ }^{3}$
}

\section{Resumo}

Foi realizado um inquérito populacional na região urbana em Montes Claros/MG, com o objetivo de verificar se há diferença na qualidade de vida entre os ativos e insuficientemente ativos e os fatores associados ao nível insuficiente de atividade física. O estudo é do tipo transversal de base populacional, sendo a amostra composta por 648 adultos de ambos os sexos. Adotouse entrevista semi-estruturada contendo variáveis socioeconômicas, demográficas e a aplicação do Questionário Internacional de Atividade Física - IPAQ - na versão curta e o SF36. Para verificar os fatores associados ao nível insuficiente de atividade física adotou-se o teste de quiquadrado $(\mathrm{p}<0,05)$ e, posteriormente o modelo de regressão logística múltipla e para verificar as diferenças no escore de qualidade de vida, foi realizado o Teste de Mann-Whitney. Os resultados demonstram prevalência do nível insuficiente de atividade física de 26,7\% [IC $95 \%$ 23,3-30,1] na população em geral. A inatividade física foi associada em indivíduos idosos, maior renda, hipertensão e baixo consumo de frutas. Além disso, o nível insuficiente de atividade física teve impacto na percepção da qualidade de vida. Conclui-se que é necessária a implantação de políticas públicas e uma educação para a saúde que possibilite uma maior adesão da população à prática de atividades físicas e consequentemente impacte no bem-estar da população em geral.

\section{Palavras-chave}

Atividade física; Qualidade de vida; Estilo de vida; Fatores de risco, Doenças crônicas.

\begin{abstract}
A survey was conducted in the urban population of Montes Claros / MG, in order to observe the differences in quality of life between the active and insufficiently active and factors associated with insufficient levels of physical activity in urban region of Montes Claros, Minas Gerais. The study is cross-sectional in population, with a sample of 648 adults of both sexes. A semi-structured interview containing socioeconomic, demographic and application of the International Physical Activity Questionnaire - IPAQ - short version and SF36. To identify factors associated with insufficient levels of physical activity adopting the chi-square test $(p<0.05)$, after adopting the multiple logistic regression model and to investigate the differences in quality of life scores using the Mann-Whitney test. The results demonstrate that prevalence of insufficient physical activity was 26.7\% [95\% CI 23.3 to 30.1] in general population. Physical inactivity was associated in elderly, higher income, hypertension, and low consumption of fruits. Furthermore, the insufficient level of physical activity had an impact on the perception of quality of life. In conclusion it is necessary to implement public policies and health education that enables greater population adherence to physical activity and consequently impact on the welfare of the general population.
\end{abstract}

\section{Keywords}

Physical Activity; Quality of life; Lifestyle; Risk factors; Chronic diseases.
Rev Bras Ativ Fis Saúde p. 562-572 DOI: http://dx.doi.org/10.12820/23171634.2012v17n6p562

1 Programa de Pós Graduação em Saúde Pública, Universidade Federal de Minas Gerais, Belo Horizonte, MG, Brasil.

2 Centro Desportivo, Universidade Federal de Ouro Preto, Ouro Preto, MG, Brasil.

3 Departamento de Clínica Médica, Universidade Estadual de Montes Claros, Montes Claros, MG, Brasil. 


\section{INTRODUÇÃO}

A inatividade física é a quarta principal causa de morte no mundo. Estamos vivendo uma pandemia de inatividade física, apesar das evidências dos benefícios da atividade física para a saúde serem divulgados desde a década de $1950^{1}$. No Brasil, as doenças crônicas não transmissíveis (DCNT) se tornaram a principal prioridade na área da saúde. Um fator importante na carga de doenças crônicas no Brasil é o aumento da prevalência de diabetes e hipertensão, paralelamente ao aumento da prevalência de excesso de peso. Esses aumentos estão associados principalmente a mudanças desfavoráveis na dieta e na atividade física entre os brasileiros ${ }^{2}$.

Além de ser um dos principais fatores de risco modificáveis para as doenças crônicas, a atividade física influencia positivamente o bem-estar ${ }^{3,4}$. Entretanto, o fenômeno da adesão a atividade física é complexo e multifatorial ${ }^{1}$ Embora seja crescente o número de pesquisas realizadas no Brasil sobre o tema ${ }^{5}$, ainda são poucos os estudos baseados em uma amostragem populacional nas últimas décadas ${ }^{6,7,8}$.

A promoção da atividade física é uma das prioridades da Estratégia Global para a Promoção da Alimentação Saudável, Atividade Física e Saúde da Organização Mundial de Saúde 9 . Os objetivos desta estratégia é o desenvolvimento, fortalecimento e implementação de políticas globais, nacionais e regionais para a adoção de hábitos saudáveis pela população ${ }^{10}$.

Com o intuito de se verificar a prevalência da inatividade física em indivíduos de uma região urbana, foi escolhida a cidade de Montes Claros. Esta ocupa posição de centralidade na região norte de Minas Gerais, constituindo o núcleo urbano mais expressivo dessa.

O município de Montes Claros vive uma transição demográfica e epidemiológi$\mathrm{ca}^{10}$. O índice de envelhecimento na população em Montes Claros em 1980 era de $6,5 \%$ e aumentou para $14,3 \%$ em 2001 . Além disso, quando verificada a mortalidade proporcional por causa básica referente aos anos de 1996-2005, verifica-se que há preponderância de óbitos por doenças do aparelho circulatório seguido de neoplasias ${ }^{10}$.

Considerando a importância da prática regular da atividade física na prevenção e/ou tratamento de determinadas DCNT e, sua influência para se obter uma vida com melhor qualidade, o objetivo do presente estudo foi verificar se há diferença na qualidade de vida entre os indivíduos ativos e insuficientemente ativos e os fatores associados ao nível insuficiente de atividade física na região urbana de Montes Claros/Minas Gerais.

\section{METODOLOGIA}

O estudo é do tipo transversal de base populacional. Os dados foram coletados no período de fevereiro a agosto de 2008, por entrevistadores treinados. Foram realizadas entrevistas face a face, com a utilização de questionário semi estruturado. $\mathrm{O}$ modelo de amostragem foi por conglomerados.

Segundo o recenseamento do censo do Instituto Brasileiro de Geografia e Estatística (IBGE, 2000) ${ }^{10}$, Montes Claros apresenta uma população residente de 306.947 habitantes no município (cerca de $21 \%$ do total regional), sendo destes 289.183 na região urbana e 17.764 na região rural. $\mathrm{O}$ número total de pessoas maiores de 18 anos residentes na zona urbana é de 213.711 e o número total de domicílios urbanos é de 71.041.

O município de apresenta 216 setores censitários. Entretanto, foram retirados da seleção os setores não-urbanos (rurais) e urbanos especiais (asilos, creches, orfanatos, 
hospitais, conventos) que totalizavam 35. A amostra foi constituída de 181 setores censitários, 163 são "urbanos não especiais" (96\% da população urbana) e 18 "urbanos subnormais" (aglomerados, favelas; $4 \%$ da população urbana). Para a representatividade dos setores censitários urbanos, dentre os 181 setores censitários foram sorteados 17. Destes, 15 "urbanos não especiais" e dois "urbanos subnormais".

Dentro de cada setor foi sorteado um quarteirão, iniciando a coleta na primeira rua do lado direito. Os domicílios foram amostrados em ordem de três, sendo o primeiro de cada três visitado. Quando não foi possível realizar a entrevista, o domicílio seguinte era visitado e a contagem reiniciada deste ponto. Após o termino da rua os entrevistadores viravam a direita seguindo o mapa de cada setor censitário. Em cada domicílio foi entrevistado um morador, sendo a escolha do entrevistado realizado por sorteio aleatório simples.

Este estudo faz parte de uma dissertação de mestrado denominada "Qualidade de vida e fatores de risco para as doenças crônicas não transmissíveis em Montes Claros, Minas Gerais". O tamanho da amostra foi calculado para uma prevalência estimada do fator de risco excesso de peso de 30\% ( $p=0,3)$. Optou-se por eleger a prevalência do sobrepeso por ser este um dos fatores de risco de maior prevalência para as DCNT. Assim, para um intervalo de confiança de 95\% ( $\mathrm{z}=1,96)$ um erro fixado de $5 \%$ (d), e um efeito de delineamento (deff) de 30\% (deff = 1.3), o tamanho da amostra inicial seria de 422 pessoas $n=\left(z^{2} \cdot p \cdot(1-p) \cdot d e f f / d^{2}\right)$. A verificação do efeito do delineamento (deff $=1,3)$ foi realizada comparando-se a variância estimada pelo processo sob conglomerado (cong.) àquela que seria obtida, caso o procedimento adotado fosse o da amostragem casual simples (acs); A amostra piloto forneceu uma variância (cong.) $=0,00062444$ e variância (acs) $=0,00048198$ cujo deff foi estimado em 1,3 (0,00062444/0,00048198). Assim, o tamanho de amostra foi igualado a 648 .

Para mensurar o nível de atividade física foi adotada a versão curta do Questionário Internacional de Atividade Física (IPAQ ${ }^{11}$. O IPAQé um questionário que permite estimar o tempo semanal gasto em atividades físicas de intensidade moderada e/ou vigorosa, em diferentes contextos do cotidiano, como, trabalho, deslocamento ativo, tarefas domésticas e lazer, e ainda o tempo despendido em atividades passivas, realizadas na posição sentada. O IPAQ curto é composto por sete questões abertas e suas informações permitem estimar o tempo despendido por semana, em diferentes dimensões de atividade física (caminhadas e esforços físicos de intensidades moderada e vigorosa) e de inatividade física (posição sentada).

A classificação do nível de atividade física foi feita considerando o tempo total dos quatro domínios estipulados pelo instrumento. Os indivíduos que praticavam 150 minutos ou mais de atividade física semanal foram considerados ativos, os que somaram um tempo menor que 150 minutos de foram considerados insuficientemente ativos (irregularmente ativos e/ou inativos).

Foram avaliados os fatores socioeconômicos: sexo; cor da pele (auto definida pelos entrevistados segundo as classificações do IBGE: parda, negra, branca, amarela e indígena); idade em faixas etárias (18 a 40; 41 a 64; $\geq 65$ ); união consensual (casado/união estável, solteiro, separado, divorciado, viúvo); escolaridade em anos (zero a oito; nove a $11 ; \geq 12$ ); renda familiar em salários mínimos (menor que três salários; entre três e seis; maior que seis); atividade remunerada (se atuante no mercado de trabalho formal ou informal; aposentado, dona de casa, estudante, etc.).

A presença de hipertensão arterial auto-referida foi avaliada através da pergunta: "Algum médico já disse que o Sr. (Sra.) tem hipertensão arterial?". O consumo de frutas, verduras e legumes foi verificado pelo questionário de frequência alimentar ${ }^{12}$. Foi considerado baixo consumo a ingestão menor que uma vez ao dia. 
Para a avaliação da Qualidade de Vida foi utilizado o questionário The Medical Outcomes Study (SF-36) ${ }^{13}$. O SF-36 é um questionário multidimensional que engloba oito domínios, formado por 36 itens, compreendido: 1. Capacidade Funcional (CF), investiga a presença e extensão das dificuldades relacionadas a atividades diárias como subir escadas, tomar banho, etc., com dez itens; 2. Limitações por Aspectos Físicos (AF), pesquisa limitações e o quanto essas limitações dificultam nas tarefas diárias ou trabalho, com quatro itens; 3. Dor (DR), investiga a presença de sofrimento, fundamentando-se na intensidade da dor, extensão ou interferência nas atividades diárias, com dois itens; 4. Estado Geral de Saúde (EGS), são aspectos gerais relacionados à percepção de saúde, com cinco itens; 5. Vitalidade (VT) considera o nível de vigor, energia e cansaço, com quatro itens; 6. Aspectos Sociais (AS) demonstra o quanto um problema físico ou emocional interfere nas atividades sociais, com dois itens; 7. Aspectos Emocionais (AE), investiga a participação em atividades de trabalho e tempo dispensado em decorrência de problemas emocionais, com três itens; 8. Saúde Mental (SM), investiga a presença de ansiedade, depressão, alterações do comportamento, descontrole emocional e bem-estar psicológico, com cinco itens. Além destes componentes, o questionário engloba mais uma questão de avaliação comparativa entre as condições de saúde atual e de um ano atrás que não entra na pontuação de nenhuma das oito dimensões.

Para a avaliação dos resultados desse instrumento, após sua aplicação, é atribuído um valor a cada questão, o qual, posteriormente, é transformado num escore de 0 a 100, onde 0 corresponde a um pior estado de saúde, e 100 a um melhor, sendo analisada cada dimensão em separado. Não existe um único valor que resuma toda a avaliação, e que corresponda a um estado geral de saúde melhor ou pior.

\section{Análise estatística}

O teste do qui-quadrado foi adotado para verificar diferenças na proporção de inatividade física segundo as categorias das variáveis independentes. Foram consideradas estatisticamente significativas aquelas diferenças que apresentaram valor de $p \leq 0,25$ para a inclusão no modelo de regressão logística múltipla. A variável dependente foi o nível insuficiente de atividade física e as variáveis independentes foram associação dos fatores socioeconômicos, a presença de co-morbidade e dieta. O modelo final foi composto somente pelas variáveis que apresentaram valor $\mathrm{p}<0,05$ e foram construídas as estimativas de razão de chances (OR) e intervalos com 95\% de confiança (IC 95\%).

Em relação à qualidade de vida, foi realizada uma análise descritiva simples como cálculo de medida de tendência central e dispersão entre as categorias no nível de atividade física. Em seguida, realizou-se análise bivariada através do teste não paramétricos Mann-Whitney. Foram considerados significativos os valores de p menores que 0,05. As análises foram realizadas no software SPSS versão 17.0.

O estudo foi aprovado pelo Comitê de Ética em Pesquisa da Universidade Estadual de Minas Gerais, processo no 677 . Todos os participantes assinaram um termo de consentimento livre e esclarecido, após concordarem em participar da pesquisa.

\section{RESULTADOS}

Entre a população estudada, a presença de mulheres foi maior em relação aos homens, a média geral de idade é de 43 anos. Houve maior predominância de cor parda ou negra (61,7\%), presença de união estável $(56,6 \%)$ e ausência de atividade remunerada (60,8\%). A maioria dos entrevistados tem baixa escolaridade e renda (Tabela 1). 
Tabela 1 - Características sócio-econômicas em relação ao nível de atividade física na população da região urbana de Montes Claros, Minas Gerais, em 2008 ( $n=648)$.

\begin{tabular}{|c|c|c|c|c|c|}
\hline \multirow[b]{2}{*}{ Variáveis } & \multicolumn{5}{|c|}{ Nível de atividade física } \\
\hline & & $\mathrm{n}$ & Total (\%) & $\begin{array}{l}\text { Insuficientemente } \\
\text { Ativos (\%) }\end{array}$ & Valor $p$ \\
\hline \multirow{2}{*}{ Sexo } & Masculino & 170 & 26,2 & 31,8 & \multirow{2}{*}{0,08} \\
\hline & Feminino & 478 & 73,8 & 24,9 & \\
\hline \multirow{3}{*}{ Idade (anos) } & 18 a 40 & 284 & 43,8 & 21,8 & \multirow{3}{*}{$<0,00^{*}$} \\
\hline & 41 a 64 & 308 & 47,5 & 25,6 & \\
\hline & $\geq 65$ & 56 & 8,6 & 57,1 & \\
\hline \multirow{2}{*}{ Cor da pele** } & Parda/Negra & 400 & 61,7 & 15,4 & \multirow{2}{*}{$0,03 *$} \\
\hline & Branca/Outras & 248 & 38,3 & 11,3 & \\
\hline \multirow[b]{2}{*}{ União Estável *** } & Sim & 367 & 56,6 & 25,3 & \multirow{2}{*}{0,37} \\
\hline & Não & 281 & 43,4 & 28,5 & \\
\hline \multirow[b]{2}{*}{ Atividade Remunerada**** } & Sim & 254 & 39,2 & 24,4 & \multirow{2}{*}{0,29} \\
\hline & Não & 394 & 60,8 & 28,2 & \\
\hline \multirow{3}{*}{ Escolaridade (anos) } & 0 a 8 & 298 & 46,0 & 28,5 & \multirow{3}{*}{0,07} \\
\hline & 9 a 11 & 257 & 39,7 & 22,2 & \\
\hline & $\geq 12$ & 93 & 14,4 & 33,3 & \\
\hline \multirow{3}{*}{$\begin{array}{l}\text { Renda } \\
\text { (salários mínimos)***** }\end{array}$} & $<3$ & 465 & 71,8 & 26,2 & \multirow{3}{*}{0,08} \\
\hline & 3 a 6 & 113 & 17,4 & 25,5 & \\
\hline & $>6$ & 70 & 10,8 & 39,3 & \\
\hline \multirow{2}{*}{$\begin{array}{l}\text { Hipertensão Arterial } \\
\text { auto-referida }\end{array}$} & Não & 437 & 67,4 & 21,7 & \multirow{2}{*}{$<0,01^{*}$} \\
\hline & $\operatorname{Sim}$ & 211 & 32,5 & 36,9 & \\
\hline \multirow[t]{2}{*}{ Baixo consumo de Fruta } & Sim & 595 & 91,8 & 43,4 & \multirow{2}{*}{$<0,01^{*}$} \\
\hline & Não & 53 & 8,1 & 25,2 & \\
\hline \multirow{2}{*}{ Baixo consumo de Verdura } & Não & 557 & 85,9 & 26,7 & \multirow{2}{*}{0,94} \\
\hline & Sim & 91 & 14,0 & 26,3 & \\
\hline
\end{tabular}

* Valor de $\mathrm{p} \leq 0,05$.

* Classificado conforme os critérios do IBGE no Censo Demográfico 2000, sendo agrupado os indivíduos auto-denominado por cor da pele parda e negra e os demais foram classificados como outras; *** Classificado como união estável (sim); solteiro, viúvo, divorciado (não);

**** Classificado como atuante no mercado de trabalho formal ou informal (sim); aposentado, dona de casa, estudante, etc. (não);

**** Salário vigente na época - R\$ 415,00

A prevalência de insuficientemente ativos na população adulta da região urbana de Montes Claros é de 26,7\% (IC95\% 23,3-30,1). A prevalência de inatividade física insuficiente aumentou com a idade, sendo de $21,8 \%$ na faixa etária de 18 a 40 anos, 25,6\% entre 41 a 60 anos e 57,1\% nos idosos ( $\geq$ de 65 anos). A cor parda e negra teve uma prevalência de atividade física insuficiente de $15,4 \%$ e $11,3 \%$ respectivamente, em relação às outras. A prevalência de atividade física insuficiente entre os hipertensos foi de $36,9 \%$, entre os que relataram baixo consumo de frutas de $43,8 \%$ e baixo consumo de verduras de $26,9 \%$.

$\mathrm{Na}$ análise univariada a inatividade física apresentou-se positivamente associada entre os idosos $\mathrm{OR}=2,61$ (IC95\%1,70-4,01), renda maior que seis salários mínimos OR=1,82 (IC95\% 1,04-3,20), presença de hipertensão arterial OR-2,27 (IC95\% 1,28-4,0) e baixo consumo de frutas OR=2,11 (1,47-3,0) (tabela 2). 
Tabela 2 - Fatores associados ao nível insuficiente de atividade física na população da região urbana de Montes Claro, medido através de regressão logística.

\begin{tabular}{|c|c|c|c|}
\hline Variáveis & & $\begin{array}{c}\text { Razão de Chances (OR) } \\
\text { (OR Bruto - IC95\%) }\end{array}$ & $\begin{array}{l}\text { Razão de Chances (OR) } \\
\text { (OR ajustado -\#IC95\%) }\end{array}$ \\
\hline \multirow[t]{3}{*}{ Idade (anos) } & 18 a 40 & 1 & 1 \\
\hline & 41 a 64 & $1,23(0,84-1,80)$ & $0,84(0,53-1,34)$ \\
\hline & $\geq 65$ & $2,61(1,70-4,01)$ & $2,47(1,20-3,07)$ \\
\hline \multirow[t]{2}{*}{ Cor da pele** } & Branca & 1 & \\
\hline & Parda/Preta & $0,56(0,32-1,00)$ & \\
\hline \multirow[t]{3}{*}{ União Consensual*** } & Outras & 1 & \\
\hline & Sim & & \\
\hline & Não & $1,17(0,82-1,66)$ & \\
\hline \multirow{2}{*}{$\begin{array}{l}\text { Atividade } \\
\text { Remunerada**** }\end{array}$} & Sim & 1 & \\
\hline & Não & $1,21(0,84-1,74)$ & \\
\hline \multirow{3}{*}{ Escolaridade (anos) } & 0 a 8 & 1 & \\
\hline & 9 a 11 & $0,71(0,48-1,08)$ & \\
\hline & $\geq 12$ & $1,25(0,76-2,06)$ & \\
\hline \multirow{3}{*}{$\begin{array}{l}\text { Renda } \\
\text { (salários } \\
\text { mínimos)**** }\end{array}$} & $<3$ & 1 & 1 \\
\hline & 3 a 6 & $0,96(0,58-1,60)$ & $1,15(0,67-1,95)$ \\
\hline & $>6$ & $1,82(1,04-3,20)$ & $1,95(1,09-3,51)$ \\
\hline \multirow[t]{2}{*}{ Hipertensão arterial } & Não & 1 & 1 \\
\hline & Sim & $2,27(1,28-4,0)$ & $1,83(1,15-2,92)$ \\
\hline \multirow{2}{*}{$\begin{array}{l}\text { Baixo consumo de } \\
\text { fruta }\end{array}$} & Não & 1 & 1 \\
\hline & Sim & $2,11(1,47-3,0)$ & $2,0(1,04-3,9)$ \\
\hline
\end{tabular}

\#ajustado por sexo

* Valor de $\mathrm{p} \leq 0,05$.

* Classificado conforme os critérios do IBGE no Censo Demográfico 2000, sendo agrupado os indivíduos auto-denominado por Cor da pele parda e negra e os demais foram classificados como outras;

*** Classificado como união estável (sim); solteiro, viúvo, divorciado (não);

*** Classificado como atuante no mercado de trabalho formal ou informal (sim); aposentado, dona de casa, estudante, etc. (não);

***** Salário vigente na época - R\$ 415,00

$\mathrm{Na}$ análise multivariada por meio da regressão logística, verificou-se que a atividade física insuficiente foi positivamente associada com idade, renda, hipertensão arterial e com baixo consumo de frutas, sendo ajustada por sexo. Os idosos ( $\geq$ 65 anos) apresentam uma chance de ter nível insuficiente de atividade física de 2,47 vezes (IC95\%1,20-3, Na 07) vezes em relação aos indivíduos na faixa etária de 18 a 40 anos. Quanto à renda, indivíduos com renda maior que seis salários mínimos apresentaram uma chance de ter nível insuficiente de atividade física de 1,95 vezes (IC95\%1,09-3,51) em relação aos que relataram ter uma renda menor que três salários mínimos. A presença de hipertensão arterial aumentou a chance de nível insuficiente de atividade física em 1,83 vezes (IC95\%1,15-2,92) e, a ter baixo consumo de frutas em 2.0 vezes (IC95\%1.04-3,9).

Quando observamos a qualidade de vida, a média dessa variável é menor nos indivíduos que tem nível insuficiente de atividade física em relação aos ativos nos domínios Capacidade Funcional (82,5 vs. 74,7), Limitação por Aspectos Físicos (77,6 vs. 68,9$)$, Vitalidade (66,8 vs. 62,8$)$ e Aspectos Emocionais (77,2 vs. 71,0$)$. Os maiores impactos na qualidade de vida do nível insuficiente de atividade física foram domínios Capacidade Funcional e na Limitação por Aspectos Físicos (tabela 3). 
Tabela 3 - Média e desvio padrão (DP) dos escores das dimensões do SF-36 entre os indivíduos ativos versus insuficientemente ativos na população da região urbana de Montes Claros, Minas Gerais $(n=648), 2008$.

\begin{tabular}{lccc}
\hline & $\begin{array}{c}\text { Ativos } \\
\text { Média } \pm \mathrm{DP}\end{array}$ & $\begin{array}{c}\text { Insuficientemente Ativos } \\
\text { Média } \pm \mathrm{DP}\end{array}$ & Valor $\mathrm{p}^{\mathrm{a}}$ \\
\hline Capacidade Funcional & $82,5 \pm 22,0$ & $74,7 \pm 29,0$ & $0,01^{*}$ \\
\hline Limitação por Aspectos Físicos & $77,6 \pm 38,0$ & $68,9 \pm 43,7$ & $0,02^{*}$ \\
\hline Dor & $65,8 \pm 27,9$ & $65,8 \pm 29,6$ & 0,93 \\
\hline Estado Geral de Saúde & $68,5 \pm 21,2$ & $65,3 \pm 22,0$ & 0,11 \\
\hline Vitalidade & $66,8 \pm 22,0$ & $62,8 \pm 23,8$ & $0,05^{*}$ \\
\hline Aspectos Sociais & $74,4 \pm 21,8$ & $72,3 \pm 25,5$ & 0,79 \\
\hline Aspectos Emocionais & $77,2 \pm 39,9$ & $71,0 \pm 41,6$ & $0,04^{*}$ \\
\hline Saúde Mental & $67,5 \pm 21,4$ & $65,6 \pm 22,2$ & 0,33 \\
\hline
\end{tabular}

*valor $\mathrm{p} \leq 0,05 *$ Teste de Mann-Whitney

\section{DISCUSSÃO}

A prevalência de insuficientemente ativos na população de Montes Claros foi de $26,7 \%$, sendo maior entre os idosos. Esse resultado embora elevado apresenta um índice inferior aos demonstrados pelo inquérito realizado pelo VIGITEL ${ }^{14}$. No VIGITEL, a frequência de adultos que praticam o volume recomendado de atividade física no tempo livre foi de 30,3\%, sendo maior entre os homens $(39,6 \%)$ do que entre as mulheres $(22,4 \%)$. Em outro estudo, Siqueira e cols ${ }^{15}$ realizaram uma pesquisa transversal em 42 cidades em sete estados brasileiros, com uma amostra de 4.060 adultos e 4.003 idosos; identificou-se a inatividade física em $58 \%$ dos entrevistados.

Em nosso estudo encontramos uma alta prevalência de inatividade física entre os idosos $(57,1 \%)$, corroborando aos dados identificados aos de outras pesquisas que utilizaram a mesma versão do instrumento (IPAQ curto), como o próprio VIGITEL $^{14}$. Alves e cols ${ }^{6}$ investigaram a inatividade física e alguns fatores associados em 1.018 adultos e 1.010 idosos, em 10 municípios com mais de 100 mil habitantes em Pernambuco. Os autores verificaram que 68,3\% dos idosos eram fisicamente inativos.

Alguns fatores podem explicar a alta prevalência de inatividade física em idosos. Um deles é uma possível associação com a redução da capacidade funcional na velhice, que, por conseguinte, aumenta a dependência por auxílio de outras pessoas, limitando a sua autonomia para a realização de atividades da vida diária e de lazer. Outro fator é a menor renda familiar, o que interferiria na prática de alguma atividade física, especificamente no lazer, que precisasse ser paga, ou na compra de algum acessório como tênis, agasalho, dentre outros, limitando assim, a adesão à essa prática ${ }^{16,17}$.

É relevante destacar que o tipo de atividade física praticado pelas pessoas pode variar de acordo com o nível socioeconômico. Neste estudo verificamos que o maior poder aquisitivo está associado com atividade física insuficiente. Em geral, as pessoas com um maior nível socioeconômico, são mais fisicamente ativas no lazer em relação às de menor poder aquisitivo ${ }^{18}$. Entretanto, Van Lenthe e $\operatorname{cols}^{19}$ identificaram que os residentes em áreas mais pobres apresentaram maior chance de caminhar ou andar de bicicleta para fazer compras ou ir trabalhar. A maioria das pessoas neste estudo possui uma renda familiar média inferior a três salários mínimos (71,8\%), o que poderia ser uma barreira á prática de atividades físicas como meio de lazer, como apontado nos estudos. 
Outro fator que pode contribuir à adesão da população à prática da atividade física é a topografia do relevo da cidade. Montes Claros possui uma topografia plana, o que contribui para que as pessoas adquiram o hábito de se deslocarem a pé ou de bicicleta, seja como atividade de lazer e/ou de deslocamento ativo. As atividades físicas no Brasil são realizadas principalmente no deslocamento para o trabalho, no próprio trabalho e nos serviços domésticos ${ }^{14}$. Entretanto, nos países ricos, a maior parte da atividade física total ocorre no tempo de lazer ${ }^{20}$.

Encontramos associação entre a presença de HA e nível de atividade física insuficiente. Essa associação tem sido investigada por diversos pesquisadores ao longo dos anos para verificar a sua eficácia na prevenção, redução e/ou controle da HA. O clássico estudo realizado por Paffenbarger e cols ${ }^{21}$ constatou que os indivíduos que praticavam exercício físico de forma regular apresentavam risco 35\% menor de desenvolver HA do que os indivíduos fisicamente inativos. Segundo Fa$\operatorname{gard}^{22}$ a inatividade física aumenta em aproximadamente em 30\% a incidência da HA em relação aos fisicamente ativos. Outros estudos ${ }^{23,24}$ também apontam que a inatividade física constitui importante fator de risco para a ocorrência de eventos cardiovasculares e maior taxa de mortalidade em indivíduos com baixo nível de condicionamento físico.

Outro fator importante encontrado neste estudo foi a associação entre o baixo consumo de frutas e inatividade física. $\mathrm{O}$ consumo alimentar da população brasileira combina a tradicional dieta à base de arroz e feijão com alimentos com poucos nutrientes e muitas calorias; sendo que a ingestão diária de frutas, legumes e verduras está abaixo dos níveis recomendados pelo Ministério da Saúde (400 g) para mais de $90 \%$ da população ${ }^{25}$.

Segundo Ferreira e $\operatorname{cols}^{25}$ a redução do consumo de frutas e hortaliças tem merecido destaque como mudança negativa importante do padrão alimentar da população brasileira nas últimas décadas. A relação da atividade física e da alimentação com a saúde é estudada há muitos anos. Os comportamentos de risco de uma alimentação inadequada, inatividade física e obesidade geralmente co-ocorrem ${ }^{26}$. A prática de atividade física regular e uma alimentação equilibrada atuam diretamente na prevenção das DCNT.

Estudo $^{27}$ realizado para verificar o nível de atividade física e os hábitos alimentares de universitários da área de saúde, identificou um alto percentual de fisicamente inativos e de inadequação nos hábitos alimentares. Outra pesquisa também realizada com universitários ${ }^{28}$ teve como objetivo descrever a proporção de fatores de risco para doenças cardiovasculares, dando ênfase aos fatores nutricionais, em alunos da área de saúde de uma Universidade pública do Recife. Os dados demonstraram que foi encontrada a seguinte ordem de importância para a classificação dos fatores de risco analisados: tabagismo, inatividade física, excesso de peso, história familiar de hipertensão, diabetes e obesidade.

Essas informações encontradas na população mais jovem (universitários) são relevantes, pois há uma tendência em reproduzir esses mesmos hábitos na fase adulta e na terceira idade, incorrendo do surgimento das DCNT e em uma menor qualidade de vida.

Neste estudo encontramos que a qualidade de vida é impactada pelo nível de atividade física insuficiente. A qualidade de vida analisada na população participante deste estudo foi menor entre os insuficientemente ativos nos domínios Capacidade Funcional, Limitação por Aspectos Físicos, Vitalidade e Aspectos Emocionais; porém, não podemos inferir a temporalidade das associações encontradas. Entretanto, nosso resultado corrobora às associações encontradas no estudo 
longitudinal realizado na Holanda ${ }^{4}$. De acordo com Van Oostrom e cols ${ }^{4}$, passado 10 anos de acompanhamento dos participantes da pesquisa, os adultos que se tornaram fisicamente ativos relataram melhor qualidade de vida no domínio físico, Vitalidade e Saúde Geral, em relação aos adultos persistentemente inativos e que se tornaram inativos ao longo desse período. Evidências mostram que a atividade física regular preserva a Saúde em Geral, a Vitalidade e Função Social ${ }^{29,30,31}$. Assim, atividade física pode contribuir para melhora das atividades diárias em casa e no trabalho, além de melhorar o bem-estar emocional.

Investir na atividade física na população em geral impacta positivamente o bem -estar dos indivíduos. Pucci e cols ${ }^{31}$ em revisão sistemática constataram que quanto maior o nível de atividade física, melhor é a percepção de qualidade de vida em idosos e, adultos aparentemente saudáveis ou em diferentes condições de saúde.

Mota e cols ${ }^{28}$ realizaram um estudo para comparar o nível de qualidade de vida entre idosos participantes de programas formais de atividade física e não participantes, adotando o SF-36. Os resultados desse estudo permitiram concluir que a participação em programa de atividade física melhora a qualidade de vida relacionado a saúde, corroborando aos nossos resultados. Portanto, a prática de atividades físicas deve ser incentivada entre a população idosa, como meio de melhoria da capacidade funcional e da qualidade de vida.

Em relação aos fatores limitantes deste estudo, pode-se destacar uma maior proporção do sexo feminino. Mesmo os homens estando presentes no domicílio no momento da coleta dos dados, muitos não aceitaram participar. Entretanto, controlamos a variável sexo como fator de confusão na análise estatística realizada. Outro aspecto importante a ser considerado para a avaliação do nível de atividade física é a diversidade de procedimentos metodológicos adotados nas diversas pesquisas, o que dificulta a comparação entre os estudos.

\section{CONCLUSÃO}

A promoção de atividade física associada com melhores condições de vida pode impactar positivamente na melhora da qualidade de vida da população em geral, bem como na prevenção e/ou tratamento das DCNT.

Os resultados encontrados neste estudo demonstram que a atividade física insuficiente está associada as faixas etárias mais velhas, maior renda, a hipertensão arterial, ao baixo consumo de frutas e a percepção negativa da qualidade de vida. Fato esse que remete à necessidade de se incrementar políticas públicas focadas em ações estruturadas e sistematizadas (para atender tanto à população em geral, quanto ao público com as características apresentadas nesse estudo) direcionada para educação para a saúde, demonstrando a importância de se adotar hábitos de vida mais saudáveis (como os alimentares e a prática de atividades físicas regulares e orientadas). Como por exemplo, a capacitação dos profissionais da área da saúde e de ações pedagógicas que podem ser desenvolvidas nas escolas abordando essa temática de forma transdisciplinar.

Além de campanhas de conscientização, de igual importância é a viabilização de políticas públicas que oportunizem a população ter acesso a espaços e equipamentos de lazer, como a criação de ciclovias, pistas de caminhadas, academias populares, dentre outras adequações urbanísticas (iluminação, segurança, acessibilidade e transporte público) que permitam a prática de atividades físicas.

Enfim, o comportamento e o estilo de vida dos indivíduos e grupos sociais é um fenômeno complexo e largamente influenciado pelos aspectos como ambiente 
físico, socioeconômico, cultural e urbanísticos, assim, se faz necessário intervenções intersetoriais do setor público, de modo de não apenas conscientizar a população de adotar hábitos de vida saudáveis, mas também possibilitar a prática de atividades físicas de lazer.

\section{Agradecimentos}

Maryane Oliveira-Campos recebeu bolsa da Fundação de Amparo a Pesquisa de Minas Gerais - FAPEMIG durante a realização do mestrado em Ciências da Saúde na UNIMONTES. Este trabalho foi financiado pela Fundação de Amparo a Pesquisa de Minas Gerais - FAPEMIG.

\section{Contribuição dos autores}

- Maryane Oliveira-Campos - contribuiu na coleta dos dados e na redação

- Marcos Gonçalves Maciel - contribuiu na redação

- João Felício Rodrigues Neto - contribuiu na coleta dos dados e na redação

\section{REFERÊNCIAS}

1. Harold WK, Craig CL, Lambert EV, et al. The pandemic of physical inactivity: global action for public health. Lancet Physical Activity Series Working Group Volume 380, Issue 9838, 21-27 July 2012, Pages 294-305.

2. Schmidt MI, Duncan BB, Silva, GA, Menezes AM, et al. Doenças crônicas não transmissíveis no Brasil: carga e desafios atuais. www.thelancet.com

3. Oliveira-Campos M, Rodrigues Neto, JF. Doenças crônicas não transmissíveis: fatores de risco e repercussão na qualidade de vida. Revista Baiana Saúde Pública; 33(4), out-dez 2009.

4. Van Oostrom SH, Smit HA, Wendel-los GCW, et al. Adopting an Active Lifestyle During Adulthood and Health-Related Quality of Life: The Doetinchem Cohort Study. American Journal of Public Health e-View Ahead of Print.doi: 10.2105/AJPH.2012.301008

5. Hallal PC, Dumith SC, Bastos JP, et al. Evolução da pesquisa epidemiológica em atividade física no Brasil: Revisão sistemática. Revista Saúde Pública 2007;41(3):453-60.

6. Hallal PC, Victora CG, Wells JCK, Lima RC. Physical inactivity: prevalence and associated variables in Brazilian adults. Med Sci Sports Exerc 2003;38:1894-900.

7. Alves JGB, Siqueira FV, Figueiroa JN, et al. Prevalência de adultos e idosos insuficientemente ativos moradores em áreas de unidades básicas de saúde com e sem Programa Saúde da Família em Pernambuco, Brasil. Caderno Saúde Pública, Rio de Janeiro, 26(3):543-556, mar, 2010.

8. Baretta E, Baretta M, Perez KG. Nível de atividade física e fatores associados em adultos no Município de Joaçaba, Santa Catarina, Brasil. Caderno Saúde Pública, Rio de Janeiro, 23(7):1595-1602, jul, 2007.

9. World Health Organization. Physical inactivity: a global public health problem. http://www. who.int/dietphysicalactivity/factsheet_inactivity/en. 2004.

10. Oliveira-Campos M, Cerqueira MBR, Rodrigues-Neto JF. Dinâmica populacional e perfil de mortalidade no município de Montes Claros (MG). Ciência \& Saúde Coletiva. 2011. Vol 16 (Supl. 1):1303-1310.

11. Matsudo SM, Matsudo VR, Araújo T, et al. Questionário Internacional de Atividade Física (IPAQ): estudo de validade e reprodutibilidade no Brasil. Revista Brasileira de Atividade Física e Saúde 2001; 6:5-18.

12. Furlan-iebig R, Pastor-Valero M. Desenvolvimento de um questionário de freqüência alimentar para o estudo de dieta e doenças não transmissíveis. Revista Saúde Pública 2004;38(4):581-4581.

13. Ciconelli RM, Ferraz MB, Santos W, Meinão I, Quaresma MR. Tradução para a língua portuguesa e validação do questionário genérico de avaliação de qualidade de vida SF-36 (Brasil SF-36). Revista Brasileira de Reumatologia. 1999 Mai/Jun 39; 3:143-50.

14. Brasil. Ministério da Saúde. Secretaria de Vigilância em Saúde. Secretaria de Gestão Estratégica e Participativa. Vigitel Brasil 2012: vigilância de fatores de risco e proteção para doenças crônicas por inquérito telefônico / Ministério da Saúde, Secretaria de Vigilância em 
Saúde, Secretaria de Gestão Estratégica e Participativa. - Brasília: Ministério da Saúde, 2012

15. Siqueira FV, Facchini LA, Piccini RX, et al. Atividade física em adultos e idosos residentes em áreas de abrangência de unidades básicas de saúde de municípios das regiões Sul e Nordeste do Brasil. Caderno Saúde Pública, Rio de Janeiro, 24(1):39-54, jan, 2008.

16. Quadros Junior AC, Lamonato ACC, Gobbi S. Nível de escolaridade não influencia nível de atividade física em idosos. Motriz, Rio Claro, v.17 n.1, p.202-208, jan./mar. 2011.

17. Cassou ACN, Firmino RC, Santos MR, et al. Barreiras para a atividade física em idosos: Uma análise por grupos focais. Revista da Educação Física/UEM Maringá, v. 19, n. 3, p. 353-360, 3. trim. 2008

18. Siqueira FV, Facchini LA, Piccini RX, et al. Atividade física em adultos e idosos residentes em áreas de abrangência de unidades básicas de saúde de municípios das regiões Sul e Nordeste do Brasil. Caderno Saúde Pública, Rio de Janeiro, 24(1):39-54, jan, 2008.

19. Van Lenthe FJ, Brug J, Mackenbach JP. Neighbour hood inequalities in physical inactivity: the role of neighbor hood attractiveness, proximity to local facilities and safety in the Netherlands. Soc Sci Med 2005; 60(4): 763-75.

20. Martinez-Gonzalez MA, Varo JJ, Santos JL, et al. Prevalence of physical activity during leisure time in the European Union. Med Sci Sports Exerc. 2001;33(7):1142-6.

21. Paffenbarger RS, Wing AL, Hyde RT. Physical exercise and incidence of hyper-tension in college alumni. Am J Epidemiol 1983;117:245-57.

22. Fagard RH. Physical activity, physical fitness and the incidence of hypertension. J Hypertens 2005. 23:265-7.Rejeski, WJ, Brawley, LR., Rocha, AS. (1996). Relationships between physical activity and health-related quality of life. Exercise and Sport Sciences Reviews, 24, 71-108.

23. Myers J. Exercise and cardiovascular health. Circulation 2003;107:2-5Blacklock, R.E., Rhodes, R.E, Brown, S G. (2007). Relationship between regular walking, physical activity, and healthrelated quality of life. Journal of Physical Activity and Health, 4, 138-152.

24. Monteiro MF, Sobral Filho DC. Exercício físico e o controle da pressão arterial. Revista Brasileira de Medicina do Esporte. Vol. 10, No 6 - Nov/Dez, 2004

25. Brasil. Instituto Brasileiro de Geografia e Estatística. Pesquisa de Orçamentos Familiares. 2008-2009. Análise da Disponibilidade Domiciliar de Alimentos e do Estado Nutricional no Brasil. Ministério da Saúde. Ministério do Planejamento, Orçamento e Gestão. 2010.

26. Ferreira, A, Chiara VL, Kuschini MCC. Alimentação saudável na adolescência: consumo de frutas e hortaliças entre adolescentes brasileiros. Revista Adolescência \& Saúde, v. 4, no 2 abril 2007.

27. Marcondelli P, Costa THM, Schmitz BAS. Nível de atividade física e hábitos alimentares de universitários do $3^{\circ}$ ao $5^{\circ}$ semestres da área da saúde. Revista Nutrição, Campinas, 21(1):39-47, jan./fev., 2008.

28. Petribú MMV, Cabral PC, Arruda IKG. Estado nutricional, consumo alimentar e risco cardiovascular: um estudo em universitários. Revista Nutrição vol.22, no.6, Campinas, Nov./ Dec. 2009.

29. Mota J, Ribeiro JL, Carvalho J, Matos MG. Atividade física e qualidade de vida associada à saúde em idosos participantes e não participantes em programas regulares de atividade física. Revista Brasileira de Educação Física e Esporte. São Paulo, v.20, n.3, p.219-25, jul./set. 2006.

30. Silva RS, Silva I, Silva RA, et al. Atividade física e qualidade de vida. Ciência \& Saúde Coletiva, 15(1):115-120, 2010.

31. Rejeski, W. J., Brawley, L. R., \& Rocha, A. S. (1996). Relationships between physical activity and health-related quality of life Exercise and Sport Sciences Reviews, 24, 71-108.

32. Pucci GCMF, Rech CR, Fermino RC, Reis RS. Associação entre atividade física e qualidade de vida em adultos. Revista Saúde Pública, 2012;46(1):166-79.

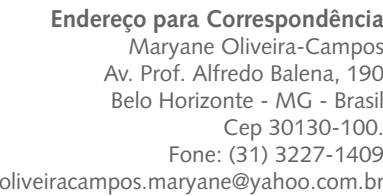

